\title{
III
}

\section{PULMONARY TUBERCULOSIS AND SYPHILIS}

\author{
By R. R. TRAIL
}

MEDICAL opinion is still strongly divided into two camps on the question of the incidence and extent of pulmonary syphilis. Some writers say that it is so rare that it can be counted as non-existent, while others call by this name a great many pulmonary conditions of indeterminate origin. It has been described as of three different forms : (I) Bronchitis in secondary syphilis; (2) Definite demonstrable gummata; and (3) A diffuse pulmonary fibrosis said to occur in the tertiary type.

The final proof must always be the demonstration of the organism in the lesions or their products, but as in lung cases such proof is almost always missing, definite diagnosis can never be established as it can be in tuberculosis, and therefore opinion has been founded mainly on the anatomical distribution together with the clinical picture and the therapeutic proof.

The post-mortem findings in various syphilitics have ranged from definite gummata to fibrosis, gummatouslike infiltrations and fibrotic cavities commonly found in conditions much more frequent than pulmonary syphilis. Later a film will be shown of a patient in whom no definite proof of either pulmonary tuberculosis or syphilis could be found, but in whom post-mortem definite syphilis of the lung was stated to be present by Professor Rössell, of Berlin.

Our steadily growing knowledge of pathology, combined with radiological findings during life, make it possible to say with a certain amount of dogmatism that there are lung lesions which appear to come within the category of gummata or gummatous sclerosis. Such lesions are, however, common to several other conditions, especially tuberculosis, so that while in a known case of syphilis some indeterminate lung condition may be by deduction considered to have such a lesion as a result of 


\section{BRITISH JOURNAL OF VENEREAL DISEASES}

his syphilis, we must admit that a syphilitic may have similar physical findings and X-ray appearances due to conditions that are non-syphilitic ; for example, he could become definitely tuberculous or have a coincident pulmonary abscess. This is why most radiologists will contend that there are no essential characteristics and that diagnosis in such cases rests on elimination of the other possible causes.

There is, of course, the further proof of improvement by treatment, but even this is not completely satisfactory as other conditions of the lung, for example, amœbic disease, are known to be amenable to certain anti-syphilitic measures and we are left with the opinion that the diagnosis may in many cases be made with great probability but never with absolute certainty.

Nevertheless, in spite of these apparent contradictions, there does seem to be a fairly definite radiological finding in lung films of certain cases of syphilis, and when it is present a positive Wassermann reaction is not unusual. This consists of a marked central fibrosis extending from the hilum outwards to the periphery in fan-shaped distribution and nearly always equally marked on both sides. This distribution is not like that of the ordinary pulmonary fibrosis of an unresolved pneumonia, nor like the basal fibrosis commonly found in long-standing chronic bronchitis and unresolved broncho-pneumonia or bronchiectasis, which, even if it is central, is almost always mainly basal and very seldom equal in its distribution in both lungs. In the same way it differs from the fibrosis of pulmonary tuberculosis which, even if bilateral, is almost always most heavily marked in the upper zones and very seldom seen with such clear distinction in both middle zones at the same time. Nor is it like the more general distribution of the various types of pneumonokoniosis, which is usually nodular or cell-nest in character. It may be found in cases of true syphilitic aortitis.

Whatever our individual beliefs may be on the existence and possibility of diagnosis of pulmonary syphilis, all of us will agree that there is a danger of missing the presence of pulmonary tuberculosis in syphilitics unless full investigation of the bronchitis in the secondary stage is undertaken, and an equal danger of missing the diagnosis of coincident syphilis in cases of pulmonary tuberculosis 
unless we suspect the extent of fibrosis present in any given case, especially where we are puzzled by the apparently abnormal resistance to a widespread tuberculous lesion.

Tuberculosis and syphilis are both comparatively common, and it follows inevitably that a certain number of patients will have both conditions. There is still, however, much discussion on the frequency of syphilis in the tuberculous, on the influence of syphilis in the evolution of tuberculosis and on the liability of the syphilitic to contract tuberculosis.

Some authors say that 3 per cent. or less of all patients with one or other condition have both; others give figures as high as I7 per cent. in recently published literature. Thus Pritchard, in a study of cases in the U.S.A., gives the figure of 3 per cent., whereas Vedder, also in the U.S.A, gives I7 per cent.

In an investigation carried out in this country from I93I to I934 of 774 patients with positive sputum, syphilis was found to be present with positive Wassermann reaction and confirmation by the patient's history in $\mathrm{II}, i . e ., \mathrm{I} \cdot 42$ per cent. These patients were from the average middle class population and excluded manual workers. Such a figure might make one think it unnecessary that a rigid search for the presence of syphilis should be carried out in all tuberculosis institutions in this country, but very little investigation on these lines has been carried out in Britain, apart from the work of Munroe, who is now inclined to think that his figures were too near the post-war years, and that of Bowman, who found in 19239.42 per cent. positives in 223 pulmonary cases. The Royal Commission on Venereal Disease in this country in I9I6 stated the percentage to be as high as from 8 to Io per cent. The conclusion would seem to be that the percentage of positive Wassermanns in tuberculosis institutions is little, if any, higher than that of the general population, but this does not contraindicate the statement already made that the presence of syphilis ought to be searched for much more commonly than is the case at present. A large number must escape notice and this applies both to the type of case who has been treated for his syphilis but does not acknowledge it, and those who have not been treated but have nevertheless a negative Wassermann reaction; the percentages of 


\section{BRITISH JOURNAL OF VENEREAL DISEASES}

these groups must, however, be roughly the same in the tuberculous and non-tuberculous.

Some writers on this subject insist, as Sergent does, that the type of tuberculosis in syphilitics is always fibroid, and that congenital syphilitics often contract tubercle which is usually fibroid in form. Further, Sergent says that the Wassermann reaction is positive in $7 \mathrm{I}$ per cent. of cases of fibroid tuberculosis and that the figures in sanatorium investigations are less only because most of the cases received into such institutions are caseous in type. With the view that the tuberculosis is fibroid in type both Gloyne and Burrell agreed when they read papers before this Society. There are two further opinions, one saying that syphilis aggravates tuberculosis and makes it caseous in type, and the second that it has no particular action. Letulle found in 58 post-mortems on pulmonary tuberculosis the same proportion of scars among the syphilitic tuberculous as in the non-syphilitic, and his findings are upheld by the researches of Kullman, Albrechts, Villers, Govaertz and Weill. It is almost impossible to come to any definite conclusion ; the problem does not lend itself to statistical investigation because it would be necessary to take into account the time at which each of the two diseases was contracted, while it is particularly difficult in many cases to say which disease was the earlier when tuberculous patients show during their illness the evidence of a primary or secondary syphilis.

Almost all observers believe that if a tuberculous patient contracts syphilis his lesions will be aggravated and his disease will become caseous in type. Dujardin and Dupres conclude by their investigations that secondary syphilis will always aggravate tuberculosis because of its inherent property of lowering the allergic resistance, while tertiary syphilis, owing to the intense allergy which accompanies it, will exercise a favourable action and produce fibrosis. Although our views on allergy in resistance against tuberculosis have varied much since these authors wrote in 1924, their theory is probably still the best explanation and certainly seems to fit actual clinical findings in cases following up over a period of years. Sergent believes, according to his lectures, that the syphilitic soil is particularly suited to the development of tuberculosis. Jacquinet holds the same view, which was 


\section{PULMONARY TUBERCULOSIS AND SYPHILIS}

that of the older French writers like Lannec. Baer and Ritte have gone so far as to say from their observations in Germany that each syphilitic is a candidate for tuberculosis, but in this country Munroe found II per cent. of hereditary syphilitics in tuberculous children and Io per cent. in non-tuberculous children, which is too small a difference to say that there can be any possible interaction between the two diseases, and Samson, in Berlin, examined 13,000 prostitutes and found amongst the syphilitic 12.5 per cent. tuberculous and amongst the nonsyphilitic I0.7 tuberculous. The conclusion would appear to be that there is no definite pre-disposition to tuberculosis amongst syphilitics.

Literature on the interplay of the two diseases is also very often contradictory. Some writers state that syphilis has a definitely unfavourable effect on the lung and that its contraction will break down previously inactive tuberculosis, but it is difficult to get absolute proof of such a statement, as we know from the records of sanatorium treatment that the chances of breakdown in uncomplicated phthisis in three to five years after discharge are as high as 50 per cent., and that many cases of uncomplicated tuberculosis may progress under the most ideal sanatorium conditions and while on complete rest. What seems to be much nearer the truth is that the type of tuberculosis found is on the average fibroid or fibrocaseous, and that the presence of long-standing syphilis has a very definite fibrosing effect, while recent syphilis well treated will have the same effect both on distribution and type of disease. The late manifestations of syphilis rarely have an adverse effect on phthisis, and usually the prognosis of cases with both diseases at this stage of syphilis is benign. The energetic treatment of recent syphilis seems to be definitely indicated, and while there is no doubt that many cases are violently upset while their anti-syphilitic treatment is being carried out the endresult justifies the means. Certainly care is necessary in the treatment of old-standing syphilis when tuberculosis intervenes, and naturally where there are already cardiovascular signs present the prognosis must inevitably be grave.

Bourgeris and Fischer believe from their researches that the average mortality among non-syphilitics is 9 per cent., while among the syphilitics it is only 3 per cent. Gallant, 
in an article published in 1929 in the American Review of Tuberculosis, quotes the after-histories of 346 patients, II6 of whom received treatment for syphilis and 230 did not. He shows that there is a marked decrease in the mortality rate from 57 per cent. to $2 \mathrm{I} \cdot 5$ per cent. where treatment for the syphilis was fully carried out, and what makes this finding all the more interesting is that the average death rate in all cases in the group of sanatoria considered was 26 per cent., showing that even where no treatment for the syphilis was carried out there was a definite lessened mortality. Consistent with the drop in mortality are the figures for the improvement in the tuberculosis, which was $20^{\circ} 9$ per cent. without treatment and 5I per cent. with it. Of far advanced cases whose syphilis was then treated 77 per cent. died; in similar cases where active and full treatment was carried out the death rate dropped to 42 per cent. within the same period.

These figures would seem to prove that syphilis coincident with tuberculosis has a very favourable influence on the type and development of the tuberculosis, and that treatment of the syphilis produces a marked improvement in the tuberculous lesion.

Observations on I4 cases diagnosed as having both diseases between the years 1927 and 1934 and followed up to date support these views. Their average period of observation is over seven years; only one has not been traced over the last three years. According to the Turban-Gerhardt classification of pulmonary tuberculosis, II of the I4 fall into Group III, one into Group II and two in Group I, and although the number as a whole is small, the results in the Group III cases are sufficiently striking to warrant certain comments and conclusions. Of these II, 7 are known to be alive and 6 of them had a full course of anti-syphilitic treatment; of the seventh it is not known definitely whether or not he had any specific treatment. Of the 4 dead, 2 were treated and 2 were not. To have 60 per cent. Group III cases alive on an average of fully seven years after a diagnosis of both diseases is a striking advance on an average result of sanatorium treatment in cases of similar extent of disease from tuberculous infiltration alone. The chances of survival five years after admission to a sanatorium are in the neighbourhood of 43 per cent. only according to I76 


\section{PULMONARY TUBERCULOSIS AND SYPHILIS}

statistical investigations carried out on the results of different institutions in this country: it seems justifiable to say that the added chance of survival is somewhere in the neighbourhood of 20 per cent. 